\title{
Microarrays of Synthetic Heparin Oligosaccharides
}

\author{
Jose L. de Paz, Christian Noti and Peter H. Seeberger*
}

Laboratory for Organic Chemistry, Swiss Federal Institute of Technology (ETH) Zurich, WolfgangPauli-Str. 10, HCI F315, 8093 Zurich, Switzerland

Table of Contents.

1. Complete Reference $2 d$

2. General Information

3. Synthesis of Compounds 4 and 9

\subsection{Synthesis of 4}

\subsection{Synthesis of 9}

\section{Mass Spectrometry Data of Glycoconjugates 10, 11 and 12}

5. Experimental Procedures for the Production of the Heparin Microarrays

\section{Additional References}

1. Complete Reference 2d: Blixt, O.; Head, S.; Mondala, T.; Scanlan, C.; Huflejt, M. E.; Alvarez, R.; Bryan, M. C.; Fazio, F.; Calarese, D.; Stevens, J.; Razi, N.; Stevens, D. J.; Skehel, J. J.; van Die, I.; Burton, D. R.; Wilson, I. A.; Cummings, R.; Wong, C-H.; Paulson, J. C. Proc. Nat. Acad. Sci. USA 2004, 101, 17033-17038.

2. General Information. All chemicals used were reagent grade and used as supplied except where noted. Dichloromethane $\left(\mathrm{CH}_{2} \mathrm{Cl}_{2}\right)$ and tetrahydrofuran (THF) were purified by a Cycle-Tainer Solvent 
Delivery System. Pyridine and triethylamine were distilled over $\mathrm{CaH}_{2}$ prior to use. Analytical thin layer chromatography (TLC) was performed on Merck silica gel $60 \mathrm{~F}_{254}$ plates $(0.25 \mathrm{~mm})$. Compounds were visualized by UV irradiation or dipping the plate in an anisaldehyde solution followed by heating. Flash column chromatography was carried out using forced flow of the indicated solvent on Fluka Kieselgel 60 (230-400 mesh). Gel filtration chromatography was carried out using Sephadex LH-20 from Amersham Biosciences.

${ }^{1} \mathrm{H}$ and ${ }^{13} \mathrm{C}$ NMR spectra were recorded on a Varian VXR-300 (300 MHz) or Bruker DRX500 (500 $\mathrm{MHz}$ ) spectrometer. High-resolution mass spectra (HR MALDI MS) were performed by the MS-service at the Laboratory for Organic Chemistry (ETH Zurich). ESI MS were run on an Agilent 1100 Series LC/MSD instrument. IR spectra were recorded on a Perkin-Elmer 1600 FTIR spectrometer. Optical rotations were measured using a Perkin-Elmer 241 polarimeter.

All aqueous solutions were made from nanopure water. Solutions used for chip hybridizations were sterile filtered through a $0.2 \mu \mathrm{m}$ syringe filter prior to use. Basic Fibroblast Growth Factor (FGF-2) and Anti-Human FGF-basic were purchased from PeproTech EC. Acidic Fibroblast Growth Factor (FGF-1) and Anti-Human FGF-acidic were purchased from Santa Cruz Biotechnology Inc. The primary AntiFGF antibodies were detected by using Goat Anti-Rabbit IgG Antibodies (Molecular Probes) labelled with Alexa Fluor 546 dye. CodeLink slides were purchased from Amersham Biosciences. Microarrays were constructed using a Perkin Elmer noncontact printer. For all incubations, $90 \mu \mathrm{L}$ of protein solution was applied to the slide by using HybriSlip Hybridization Covers from Grace BioLabs. Slides were scanned using a LS400 scanner from Tecan and quantified using Scan Array Express Software. All data are the average signal of at least 15 spots on a single array; errors are the standard deviations of those measurements.

\section{Synthesis of Monosaccharides 4 and 9.}




\subsection{Synthesis of Monosaccharide 4.}

Scheme 1. Synthesis of Monosaccharide 4
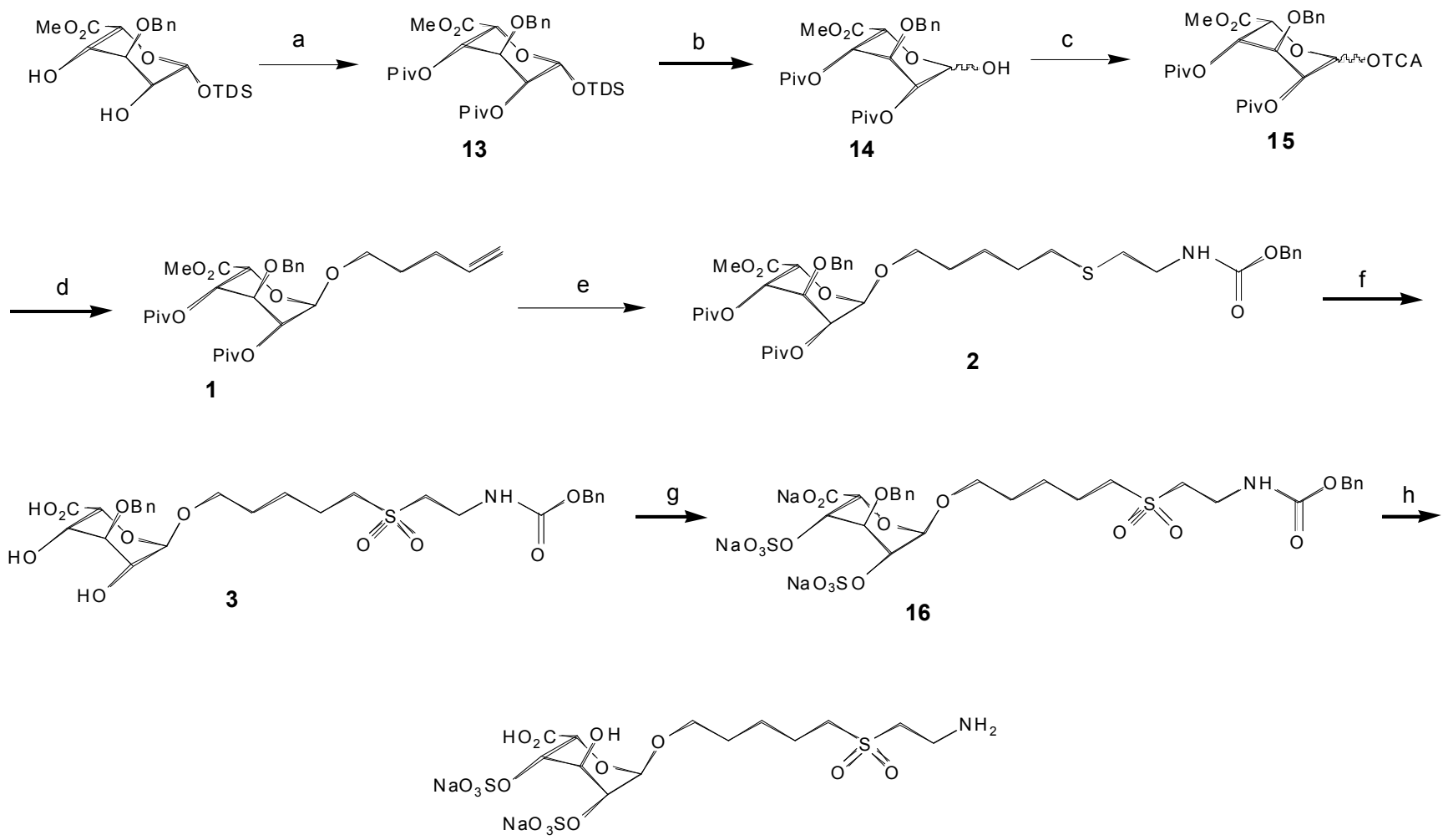

4

${ }^{a}$ Reagents and conditions: a) PivCl, Py, 93\%; b) (HF) $\cdot \mathrm{Py}, \mathrm{THF}, 80 \%$; c) $\mathrm{Cl}_{3} \mathrm{CCN}, \mathrm{K}_{2} \mathrm{CO}_{3}, 90 \%$; d) $n$ pentenyl alcohol, TMSOTf, $\mathrm{CH}_{2} \mathrm{Cl}_{2}, 97 \%$; e) $\mathrm{SH}-\mathrm{CH}_{2}-\mathrm{CH}_{2}-\mathrm{NHZ}$, AIBN, THF, $75^{\circ} \mathrm{C}, 82 \%$; f) $\mathrm{LiOH}$, $\mathrm{H}_{2} \mathrm{O}_{2}$, then $\mathrm{KOH}, 88 \%$; g) $\mathrm{SO}_{3} \cdot \mathrm{Py}, \mathrm{Py}, 85 \%$; h) $\mathrm{H}_{2}, \mathrm{Pd} / \mathrm{C}, 95 \%$.

\section{Methyl (dimethylthexylsilyl 3-O-benzyl-2,4-di- $O$-pivaloyl- $\beta$-L-idopyranoside) uronate (13).}

Methyl (dimethylthexylsilyl 3-O-benzyl- $\beta$-L-idopyranoside) uronate ${ }^{1}$ (1.04 g, 2.37 mmol) was dissolved in pyridine $(10 \mathrm{~mL})$ at room temperature. Pivaloyl chloride $(5 \mathrm{~mL})$ and a catalytic amount of DMAP were added and the mixture was stirred for $12 \mathrm{~h}$ at room temperature. The solution was diluted with $\mathrm{CH}_{2} \mathrm{Cl}_{2}(250 \mathrm{~mL})$, washed with $1 \mathrm{M}$ solution of $\mathrm{HCl}, \mathrm{H}_{2} \mathrm{O}$, dried $\left(\mathrm{MgSO}_{4}\right)$ and concentrated in vacuo. 
The residue was purified by flash chromatography (14:1 hexane/EtOAc) to afford 13 (1.30 g, 93\%). $[\alpha]^{24}=+24.0\left(c=0.58, \mathrm{CHCl}_{3}\right) ; \mathrm{IR}($ thin film on $\mathrm{NaCl}): v=2964,2862,1764,1728,1149,1046 \mathrm{~cm}^{-1}$; ${ }^{1} \mathrm{H}$ NMR $\left(300 \mathrm{MHz}, \mathrm{CDCl}_{3}\right): \delta 7.36-7.26(\mathrm{~m}, 5 \mathrm{H}), 5.15(\mathrm{~m}, 1 \mathrm{H}), 5.03(\mathrm{~m}, 2 \mathrm{H}), 4.82(\mathrm{~d}, J=11.7 \mathrm{~Hz}$, 1H), $4.74(\mathrm{~d}, J=11.7 \mathrm{~Hz}, 1 \mathrm{H}), 4.57(\mathrm{~m}, 1 \mathrm{H}), 3.81(\mathrm{~m}, 1 \mathrm{H}), 3.73(\mathrm{~s}, 3 \mathrm{H}), 1.61(\mathrm{~m}, 1 \mathrm{H}), 1.22-1.19(2 \mathrm{~s}$, $18 \mathrm{H}), 0.85(\mathrm{~m}, 12 \mathrm{H}), 0.21(\mathrm{~s}, 3 \mathrm{H}), 0.14(\mathrm{~s}, 3 \mathrm{H}) .{ }^{13} \mathrm{C} \mathrm{NMR}\left(75 \mathrm{MHz}, \mathrm{CDCl}_{3}\right): \delta$ 178.4, 178.0, 168.1, $137.5,128.7,128.2,127.9,93.8,76.1,73.23,73.18,67.6,52.2,39.2,39.1,34.1,27.5,27.4,27.2,25.1$, 20.3, 20.2, 18.7, -1.6, -3.5. HR MALDI MS: $m / z$ : calcd for $\mathrm{C}_{32} \mathrm{H}_{52} \mathrm{O}_{9} \mathrm{SiNa}$ : 631.3273; found: 631.3283 $[\mathrm{M}+\mathrm{Na}]^{+}$.

Methyl 3-O-benzyl-2,4-di- $\boldsymbol{O}$-pivaloyl- $\boldsymbol{\alpha}, \boldsymbol{\beta}$-L-idopyranosuronate (14). To a cooled solution $\left(-10^{\circ} \mathrm{C}\right)$ of 13 (1.30 g, $2.14 \mathrm{mmol})$ in anhydrous THF $(30 \mathrm{~mL})$, an excess of $(\mathrm{HF})_{\mathrm{n}} \cdot \mathrm{Py}$ complex $(6 \mathrm{~mL})$ was added. The reaction was then warmed up to $0^{\circ} \mathrm{C}$ and stirred under an argon atmosphere. After $48 \mathrm{~h}$, $\mathrm{CH}_{2} \mathrm{Cl}_{2}(75 \mathrm{~mL})$ was added and the mixture was washed with $\mathrm{H}_{2} \mathrm{O}(2 \mathrm{x} 40 \mathrm{~mL})$ and saturated $\mathrm{NaHCO}_{3}$ solution $(40 \mathrm{~mL})$ until neutral $\mathrm{pH}$ was reached. The organic layer was dried $\left(\mathrm{MgSO}_{4}\right)$ and concentrated in vacuo. The residue was purified by flash chromatography (5:2 hexane/EtOAc) to yield 14 (800 mg, $80 \%$ ) as a $\alpha / \beta$ mixture. IR (thin film on $\mathrm{NaCl}): v=3056,2985,1421,1267,892 \mathrm{~cm}^{-1} ;{ }^{1} \mathrm{H}$ NMR $(300$ $\left.\mathrm{MHz}, \mathrm{CDCl}_{3}\right): \delta$ 7.28-7.19 (m, 5H), 5.29-5.09 (m, 2H), 4.99-4.54 (m, 3H), $4.73(\mathrm{~m}, 2 \mathrm{H}), 3.91-3.78(\mathrm{~m}$, $1 \mathrm{H}), 3.66(\mathrm{~m}, 3 \mathrm{H}), 1.18-1.11(\mathrm{~m}, 18 \mathrm{H}) .{ }^{13} \mathrm{C} \mathrm{NMR}\left(75 \mathrm{MHz}, \mathrm{CDCl}_{3}\right): \delta 178.4,178.1,177.9,177.8,169.6$, $168.8,137.4,137.3,128.6,128.1,127.84,127.78,127.5,93.2,92.7,75.1,74.7,73.2,72.8,69.4,68.5$, $68.4,68.1,67.3,52.4,39.3,39.1,38.9,27.4,27.32,27.25,27.0$. HR MALDI MS: $m / z$ : calcd for $\mathrm{C}_{24} \mathrm{H}_{34} \mathrm{O}_{9} \mathrm{Na}: 489.2095$; found: $489.2104[\mathrm{M}+\mathrm{Na}]^{+}$.

$O$-(Methyl 3- $O$-benzyl-2,4-di- $O$-pivaloyl- $\alpha, \beta$-L-idopyranosyluronate) trichloroacetimidate (15). To a solution of $14(700 \mathrm{mg}, 1.50 \mathrm{mmol})$ in anhydrous $\mathrm{CH}_{2} \mathrm{Cl}_{2}(8 \mathrm{~mL}), \mathrm{Cl}_{3} \mathrm{CCN}(1.80 \mathrm{~mL}, 18.0 \mathrm{mmol})$ and $\mathrm{K}_{2} \mathrm{CO}_{3}(249 \mathrm{mg}, 1.80 \mathrm{mmol})$ were added. After stirring at room temperature for $16 \mathrm{~h}$, the mixture was then filtered off and concentrated in vacuo, and the residue was purified by flash chromatography 
over a short silica gel column (4:1 hexane/EtOAc) to yield $15(828 \mathrm{mg}, 90 \%)$ as a $\alpha / \beta$ mixture. IR (thin film on NaCl): $v=3056,2974,1733,1421,1267,1144 \mathrm{~cm}^{-1} ;{ }^{1} \mathrm{H} \mathrm{NMR}\left(300 \mathrm{MHz}, \mathrm{CDCl}_{3}\right): \delta 8.72-$ $8.70(2 \mathrm{~s}, 1 \mathrm{H}), 7.32-7.22(\mathrm{~m}, 5 \mathrm{H}), 6.36-6.22(2 \mathrm{br} \mathrm{s}, 1 \mathrm{H}), 5.25-4.72(\mathrm{~m}, 5 \mathrm{H}), 4.01-3.84(\mathrm{~m}, 1 \mathrm{H}), 3.72-$ $3.69(2 \mathrm{~s}, 3 \mathrm{H}), 1.19-1.17(\mathrm{~m}, 18 \mathrm{H}) .{ }^{13} \mathrm{C} \mathrm{NMR}\left(75 \mathrm{MHz}, \mathrm{CDCl}_{3}\right): \delta 178.0,177.9,177.7,177.5,168.2$, $167.3,160.7,160.1,137.4,137.2,128.7,128.5,128.3,128.0,127.9,127.6,95.3,94.6,90.9,90.8,75.1$, $73.6,73.4,72.8,68.4,68.2,67.9,67.7,66.8,66.4,52.6,52.5,39.1,39.0,27.44,27.37,27.32$. HR MALDI MS: $m / z$ : calcd for $\mathrm{C}_{26} \mathrm{H}_{34} \mathrm{O}_{9} \mathrm{NCl}_{3} \mathrm{Na}$ : 632.1191; found: 632.1203 [M+Na] $]^{+}$

\section{Methyl ( $\boldsymbol{n}$-pentenyl 3- $\boldsymbol{O}$-benzyl-2,4-di- $\boldsymbol{O}$-pivaloyl- $\boldsymbol{\alpha}$-L-idopyranoside) uronate (1). TMSOTf (25}

$\mu \mathrm{L}, 0.14 \mathrm{mmol})$ was added at $-25^{\circ} \mathrm{C}$, under argon, to a solution of $\mathbf{1 5}(828 \mathrm{mg}, 1.36 \mathrm{mmol})$ and pentenyl alcohol $(154 \mu \mathrm{L}, 1.50 \mathrm{mmol})$ in anhydrous $\mathrm{CH}_{2} \mathrm{Cl}_{2}(10 \mathrm{~mL})$. After $15 \mathrm{~min}$, the reaction mixture was quenched with triethylamine, concentrated and the residue was purified by flash chromatography (6:1 hexane/EtOAc) to yield $1(706 \mathrm{mg}, 97 \%) .[\alpha]^{24}=-23.7\left(c=1.0, \mathrm{CHCl}_{3}\right)$; IR (thin film on $\left.\mathrm{NaCl}\right): v=$ 2954, 2862, 1728, 1456, 1277, $1144 \mathrm{~cm}^{-1} ;{ }^{1} \mathrm{H}$ NMR (300 MHz, $\left.\mathrm{CDCl}_{3}\right): \delta 7.28-7.17(\mathrm{~m}, 5 \mathrm{H}), 5.70(\mathrm{~m}$, $1 \mathrm{H}), 5.16(\mathrm{~m}, 1 \mathrm{H}), 4.93-4.81(\mathrm{~m}, 5 \mathrm{H}), 4.71(\mathrm{~d}, J=11.7 \mathrm{~Hz}, 1 \mathrm{H}), 4.64(\mathrm{~d}, J=11.7 \mathrm{~Hz}, 1 \mathrm{H}), 3.71-3.65$ $(\mathrm{m}, 5 \mathrm{H}), 3.46(\mathrm{~m}, 1 \mathrm{H}), 2.04(\mathrm{~m}, 2 \mathrm{H}), 1.63(\mathrm{~m}, 2 \mathrm{H}), 1.11(2 \mathrm{~s}, 18 \mathrm{H}) .{ }^{13} \mathrm{C} \mathrm{NMR}\left(75 \mathrm{MHz}, \mathrm{CDCl}_{3}\right): \delta 177.6$, $177.4,169.3,138.1,137.7,128.4,127.8,127.5,115.1,98.8,74.6,72.5,68.6,68.4,68.1,67.2,52.3$, 39.0, 38.8, 30.3, 28.7, 27.3, 27.2. HR MALDI MS: $m / z$ : calcd for $\mathrm{C}_{29} \mathrm{H}_{42} \mathrm{O}_{9} \mathrm{Na}$ : 557.2721; found: $557.2729[\mathrm{M}+\mathrm{Na}]^{+}$.

\section{Methyl \{ 5-[2-(benzyloxycarbonylamino)-1-thioethyl] pentyl 3-O-benzyl-2,4-di- $O$-pivaloyl- $\alpha$-L-}

idopyranoside uronate (2). A solution of 1 (85 mg, $0.16 \mathrm{mmol})$, AIBN (5.2 mg, $32 \mu \mathrm{mol})$ and 2(benzyloxycarbonylamino)-1-ethanethiol ${ }^{2}(504 \mathrm{mg}, 2.38 \mathrm{mmol})$ in $\mathrm{THF}(5 \mathrm{~mL})$ was stirred at $75^{\circ} \mathrm{C}$ under argon atmosphere. After $5 \mathrm{~h}$, the reaction mixture was concentrated and the residue was subjected to flash chromatography $\left(6: 1 \rightarrow 4: 1\right.$ hexane/EtOAc) to afford $2(98 \mathrm{mg}, 82 \%)$. $[\alpha]^{24}=-15.9(c=0.92$, $\mathrm{CHCl}_{3}$ ); IR (thin film on NaCl): $v=2923,1723,1508,1456,1144,1051 \mathrm{~cm}^{-1} ;{ }^{1} \mathrm{H} \mathrm{NMR}(300 \mathrm{MHz}$, 
$\left.\mathrm{CDCl}_{3}\right): \delta 7.37-7.26(\mathrm{~m}, 10 \mathrm{H}), 5.21(\mathrm{~m}, 1 \mathrm{H}), 5.14-5.10(\mathrm{~m}, 3 \mathrm{H}), 4.97(\mathrm{br} \mathrm{s}, 1 \mathrm{H}), 4.93(\mathrm{~m}, 1 \mathrm{H}), 4.87(\mathrm{~m}$ $1 \mathrm{H}), 4.78(\mathrm{~d}, J=11.7 \mathrm{~Hz}, 1 \mathrm{H}), 4.72(\mathrm{~d}, J=11.7 \mathrm{~Hz}, 1 \mathrm{H}), 3.78-3.33(\mathrm{~m}, 8 \mathrm{H}), 2.63(\mathrm{~m}, 2 \mathrm{H}), 2.45(\mathrm{~m}$, $2 \mathrm{H}), 1.60-1.40(\mathrm{~m}, 6 \mathrm{H}), 1.19(2 \mathrm{~s}, 18 \mathrm{H}) .{ }^{13} \mathrm{C} \mathrm{NMR}\left(75 \mathrm{MHz}, \mathrm{CDCl}_{3}\right): \delta 177.5,177.3,169.0,156.1$ $137.5,136.4,128.4,128.2,128.0,127.6,127.3,98.6,74.4,72.4,68.6,68.4,68.2,67.0,66.7,52.3,40.2$ $39.0,38.8,32.2,31.7,29.8,29.5,29.1,27.3,27.2,25.4,25.0$. HR MALDI MS: $m / z$ : calcd for $\mathrm{C}_{39} \mathrm{H}_{55} \mathrm{O}_{11} \mathrm{NSNa}$ : 768.3388; found: $768.3401[\mathrm{M}+\mathrm{Na}]^{+}$.

Monosaccharide 4. To a solution of $2(92 \mathrm{mg}, 0.12 \mathrm{mmol})$ in THF $(2.8 \mathrm{~mL})$ at $0^{\circ} \mathrm{C}, \mathrm{H}_{2} \mathrm{O}_{2}(30 \%, 0.95$ $\mathrm{mL})$ and a solution of $\mathrm{LiOH}(1 \mathrm{~N}, 1.6 \mathrm{~mL})$ were added. After stirring for $16 \mathrm{~h}$ at room temperature, the mixture was cooled to $0^{\circ} \mathrm{C}$ and $\mathrm{MeOH}(4.8 \mathrm{~mL})$ and a solution of $\mathrm{KOH}(3 \mathrm{~N}, 2.8 \mathrm{~mL})$ were added. After stirring for $16 \mathrm{~h}$ at room temperature, the reaction mixture was neutralized with IR-120- $\mathrm{H}^{+}$amberlite resin and then was filtered and concentrated. The residue was eluted from a Sephadex LH-20 chromatography column with $\mathrm{MeOH} / \mathrm{CH}_{2} \mathrm{Cl}_{2}$ (1:1) to afford 3 (64 mg, 88\%). ${ }^{1} \mathrm{H} \mathrm{NMR}(300 \mathrm{MHz}$, $\left.\mathrm{CD}_{3} \mathrm{OD}\right): \delta 7.38-7.25(\mathrm{~m}, 10 \mathrm{H}), 5.07(\mathrm{~s}, 2 \mathrm{H}), 4.89$ (br s, $\left.1 \mathrm{H}\right), 4.72-4.59(\mathrm{~m}, 3 \mathrm{H}), 4.06$ (br s, 1H), 3.77$3.70(\mathrm{~m}, 3 \mathrm{H}), 3.57-3.46(\mathrm{~m}, 3 \mathrm{H}), 3.22-3.18(\mathrm{~m}, 2 \mathrm{H}), 2.96-2.91(\mathrm{~m}, 2 \mathrm{H}), 1.75-1.43(\mathrm{~m}, 6 \mathrm{H})$. ESI MS: $m / z$ : calcd for $\mathrm{C}_{28} \mathrm{H}_{37} \mathrm{O}_{11} \mathrm{NSNa}$ : 618.2; found: $618.0[\mathrm{M}+\mathrm{Na}]^{+}$.

To a solution of $\mathbf{3}(28 \mathrm{mg}, 47 \mu \mathrm{mol})$ in anhydrous pyridine $(2 \mathrm{~mL})$ was added sulphur trioxidepyridine complex $(75 \mathrm{mg}, 0.47 \mathrm{mmol})$. After stirring for $12 \mathrm{~h}$ at room temperature under an argon atmosphere, the reaction mixture was quenched with triethylamine $(0.1 \mathrm{~mL})$ and diluted with $\mathrm{MeOH}(1$ $\mathrm{mL})$ and $\mathrm{CH}_{2} \mathrm{Cl}_{2}(1 \mathrm{~mL})$. The solution was layered on the top of a Sephadex LH-20 chromatograpy column that was eluted with $\mathrm{MeOH} / \mathrm{CH}_{2} \mathrm{Cl}_{2}$ (1:1). The fractions that contained the sulphated monosaccharide were pooled and evaporated to dryness. The residue was converted into the sodium salt by elution from a column of Dowex $50 \mathrm{WX} 4-\mathrm{Na}^{+}$with $\mathrm{MeOH} / \mathrm{H}_{2} \mathrm{O}(9: 1)$ to give $16(33 \mathrm{mg}, 85 \%)$. $[\alpha]^{24}$ $=-11.6(c=2.0, \mathrm{MeOH}) ;{ }^{1} \mathrm{H}$ NMR $\left(300 \mathrm{MHz}, \mathrm{CD}_{3} \mathrm{OD}\right): \delta 7.40-7.22(\mathrm{~m}, 10 \mathrm{H}), 5.18(\mathrm{~s}, 1 \mathrm{H}), 5.08(\mathrm{~s}$, $2 \mathrm{H}), 4.78-4.66(\mathrm{~m}, 3 \mathrm{H}), 4.45-4.43(\mathrm{~m}, 2 \mathrm{H}), 3.74-3.46(\mathrm{~m}, 5 \mathrm{H}), 3.24-3.19(\mathrm{~m}, 2 \mathrm{H}), 2.96-2.91(\mathrm{~m}, 2 \mathrm{H})$, 
1.75-1.43 (m, 6H). ESI MS: $m / z$ : calcd for $\mathrm{C}_{28} \mathrm{H}_{35} \mathrm{O}_{17} \mathrm{NNaS}_{3}: 776.1$; found: 776.0 [M-2Na+H]

A solution of $16(31 \mathrm{mg}, 38 \mu \mathrm{mol})$ in $\mathrm{MeOH} / \mathrm{H}_{2} \mathrm{O}(1 \mathrm{~mL} / 0.5 \mathrm{~mL})$ was hydrogenated in the presence of $10 \% \mathrm{Pd} / \mathrm{C}$. After $24 \mathrm{~h}$, the suspension was filtered and concentrated to give $4(21 \mathrm{mg}, 95 \%)$. $[\alpha]_{\mathrm{D}}^{24}=-$ $3.1\left(c=0.75, \mathrm{H}_{2} \mathrm{O}\right) ;{ }^{1} \mathrm{H}$ NMR $\left(500 \mathrm{MHz}, \mathrm{D}_{2} \mathrm{O}\right): \delta 4.95(\mathrm{~s}, 1 \mathrm{H}), 4.67$ (br s, 1H), 4.46-4.44 (m, $\left.2 \mathrm{H}\right), 4.34$ $(\mathrm{s}, 1 \mathrm{H}), 4.06(\mathrm{~s}, 1 \mathrm{H}), 3.59(\mathrm{~m}, 1 \mathrm{H}), 3.50-3.41(\mathrm{~m}, 4 \mathrm{H}), 3.20-3.19(\mathrm{~m}, 2 \mathrm{H}), 1.71(\mathrm{~m}, 2 \mathrm{H}), 1.53(\mathrm{~m}, 2 \mathrm{H})$ $1.41(\mathrm{~m}, 2 \mathrm{H}) .{ }^{13} \mathrm{C}$ NMR $\left(125 \mathrm{MHz}, \mathrm{D}_{2} \mathrm{O}\right): \delta 175.0,98.7,82.0,74.8,73.7,68.4,66.6,52.8,48.7,33.0$ 27.9, 24.3, 21.0. ESI MS: $m / z$ : calcd for $\mathrm{C}_{13} \mathrm{H}_{24} \mathrm{O}_{15} \mathrm{NS}_{3}$ : 530.0; found: 530.0 [M-2Na+H] .

\subsection{Synthesis of Monosaccharide 9.}

Scheme 2. Synthesis of Monosaccharide 9
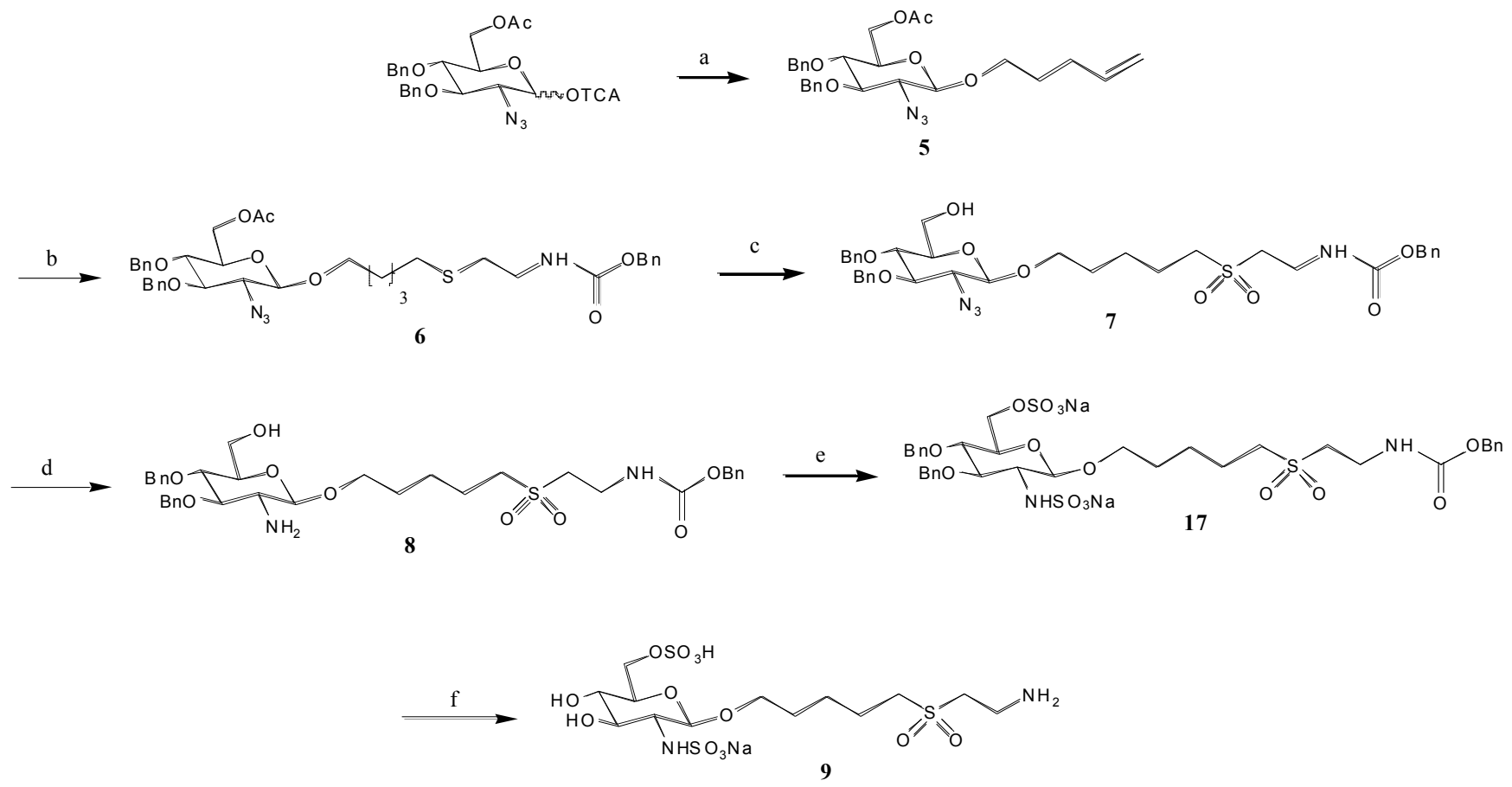

${ }^{a}$ Reagents and conditions: a) $n$-pentenyl alcohol, TMSOTf, $\mathrm{CH}_{2} \mathrm{Cl}_{2}, 65 \% \beta$ anomer; b) $\mathrm{HS}-\mathrm{CH}_{2}-\mathrm{CH}_{2}-$ NHZ, AIBN, THF, $75^{\circ} \mathrm{C}, 95 \%$; c) $\mathrm{LiOH}_{2} \mathrm{H}_{2} \mathrm{O}_{2}$; $\mathrm{KOH}, \mathrm{MeOH}$; $\mathrm{KHSO}_{5}, \mathrm{MeOH}, 88 \%$; d) $\mathrm{PMe}_{3}, \mathrm{THF}$ $\mathrm{NaOH}, 96 \%$; e) $\mathrm{SO}_{3} \cdot \mathrm{Py}, \mathrm{Py}, 84 \%$; h) $\mathrm{H}_{2}, \mathrm{Pd} / \mathrm{C}, 99 \%$.

$n$-Pentenyl 6- $\boldsymbol{O}$-acetyl-2-azido-3,4-di- $\boldsymbol{O}$-benzyl-2-deoxy- $\boldsymbol{\beta}$-D-glucopyranoside (5). TMSOTf (50 $\boldsymbol{\mu L}$ of a $0.50 \mathrm{M}$ solution in anhydrous $\left.\mathrm{CH}_{2} \mathrm{Cl}_{2}\right)$ was added at $-25^{\circ} \mathrm{C}$, under argon, to a solution of $O-(6-O-$ 
De Paz, Noti and Seeberger, Supporting Information

acetyl-2-azido-3,4-di- $O$-benzyl-2-deoxy- $\alpha, \beta$-D-glucopyranosyl) trichloroacetimidate ${ }^{3}$ (286 mg, 0.50 mmol) and $n$-pentenyl alcohol $(103 \mu \mathrm{L}, 1.00 \mathrm{mmol})$ in dry $\mathrm{CH}_{2} \mathrm{Cl}_{2}(2 \mathrm{~mL})$. After $30 \mathrm{~min}$, the reaction mixture was quenched with triethylamine, concentrated and the residue was purified by flash chromatography $(24: 1$ toluene/EtOAc) to yield $5(162 \mathrm{mg}, 65 \%)$. IR (thin film on $\mathrm{NaCl}): v=3005$, 2872, 2102, 1739, 1359, $1072 \mathrm{~cm}^{-1} ;{ }^{1} \mathrm{H}$ NMR (300 MHz, $\left.\mathrm{CDCl}_{3}\right): \delta 7.41-7.26(\mathrm{~m}, 10 \mathrm{H}), 5.83(\mathrm{~m}, 1 \mathrm{H})$, 5.10-4.79 (m, 5H), $4.58(\mathrm{~d}, J=10.8 \mathrm{~Hz}, 1 \mathrm{H}), 4.37-4.19(\mathrm{~m}, 3 \mathrm{H}), 3.93(\mathrm{~m}, 1 \mathrm{H}), 3.61-3.43(\mathrm{~m}, 5 \mathrm{H}), 2.19$ $(\mathrm{m}, 2 \mathrm{H}), 2.05(\mathrm{~s}, 3 \mathrm{H}), 1.76(\mathrm{~m}, 2 \mathrm{H}) .{ }^{13} \mathrm{C} \mathrm{NMR}\left(75 \mathrm{MHz}, \mathrm{CDCl}_{3}\right): \delta 170.9,138.1,138.0,137.7,128.78$ $128.74,128.3,128.2,115.3,102.4,83.3,77.6,75.8,75.3,73.2,69.8,66.6,63.1,30.2,28.9,21.1 . \mathrm{HR}$ MALDI MS: $m / z$ : calcd for $\mathrm{C}_{27} \mathrm{H}_{33} \mathrm{O}_{6} \mathrm{~N}_{3} \mathrm{Na}$ : 518.2267; found: $518.2274[\mathrm{M}+\mathrm{Na}]^{+}$.

\section{5-[2-(Benzyloxycarbonylamino)-1-thioethyl] pentyl 6-O-acetyl-2-azido-3,4-di- $O$-benzyl-2-deoxy-}

$\boldsymbol{\beta}$-D-glucopyranoside (6). A solution of 5 (77 mg, $0.16 \mathrm{mmol})$, AIBN (10 mg, $62 \mu \mathrm{mol})$ and 2(benzyloxycarbonylamino)-1-ethanethiol (491 mg, $2.33 \mathrm{mmol})$ in THF $(8 \mathrm{~mL})$ was stirred at $75^{\circ} \mathrm{C}$ under argon atmosphere. After $12 \mathrm{~h}$, the reaction mixture was concentrated and the residue was subjected to flash chromatography $(4: 1 \rightarrow 2: 1$ hexane/EtOAc) $)$ to afford $6(107 \mathrm{mg}, 95 \%) .[\alpha]^{24}{ }_{\mathrm{D}}=-8.1(c=0.95$, $\mathrm{CHCl}_{3}$ ); IR (thin film on $\left.\mathrm{NaCl}\right): v=3005,2944,2103,1723,1513,1072 \mathrm{~cm}^{-1} ;{ }^{1} \mathrm{H} \mathrm{NMR}(300 \mathrm{MHz}$, $\left.\mathrm{CDCl}_{3}\right): \delta 7.39-7.24(\mathrm{~m}, 15 \mathrm{H}), 5.17($ br s, $1 \mathrm{H}), 5.11(\mathrm{~s}, 2 \mathrm{H}), 4.93-4.78(\mathrm{~m}, 3 \mathrm{H}), 4.56(\mathrm{~d}, J=10.8 \mathrm{~Hz}$ $1 \mathrm{H}), 4.35-4.18(\mathrm{~m}, 3 \mathrm{H}), 3.90(\mathrm{~m}, 1 \mathrm{H}), 3.56-3.35(\mathrm{~m}, 7 \mathrm{H}), 2.66(\mathrm{~m}, 2 \mathrm{H}), 2.52(\mathrm{~m}, 2 \mathrm{H}), 2.03(\mathrm{~s}, 3 \mathrm{H})$, $1.65-1.45(\mathrm{~m}, 6 \mathrm{H}) .{ }^{13} \mathrm{C}$ NMR $\left(75 \mathrm{MHz}, \mathrm{CDCl}_{3}\right): \delta 170.9,156.5,138.0,137.7,136.7,128.8,128.3$, $128.2,102.4,83.3,77.5,75.8,75.3,73.2,70.3,67.0,66.7,63.1,40.3,32.4,31.9,29.6,29.3,25.4,21.1$. HR MALDI MS: $m / z$ : calcd for $\mathrm{C}_{37} \mathrm{H}_{46} \mathrm{O}_{8} \mathrm{~N}_{4} \mathrm{SNa}$ : 729.2934; found: 729.2945 [M+Na] $]^{+}$

Monosaccharide 9. To a solution of $6(81 \mathrm{mg}, 0.12 \mathrm{mmol})$ in $\mathrm{THF}(2.6 \mathrm{~mL})$ at $0^{\circ} \mathrm{C}, \mathrm{H}_{2} \mathrm{O}_{2}(30 \%, 0.90$ $\mathrm{mL})$ and a solution of $\mathrm{LiOH}(1 \mathrm{~N}, 1.5 \mathrm{~mL})$ were added. After stirring for $16 \mathrm{~h}$ at room temperature, the mixture was cooled to $0^{\circ} \mathrm{C}$ and $\mathrm{MeOH}(4.4 \mathrm{~mL})$ and a solution of $\mathrm{KOH}(3 \mathrm{~N}, 2.6 \mathrm{~mL})$ were added. After stirring for $16 \mathrm{~h}$ at room temperature, the reaction mixture was neutralized with $\mathrm{IR}-120-\mathrm{H}^{+}$amberlite 
De Paz, Noti and Seeberger, Supporting Information

resin and then was filtered and concentrated. The residue was dissolved in methanol $(5 \mathrm{~mL})$ at $0^{\circ} \mathrm{C}$ and $\mathrm{KHSO}_{5}(1 \mathrm{~mL}$ of a $0.35 \mathrm{M}$ solution in water) was added under stirring. After $1.5 \mathrm{~h}$, the reaction mixture was neutralized with $1 \mathrm{~N}$ solution of $\mathrm{LiOH}$, diluted with $\mathrm{CH}_{2} \mathrm{Cl}_{2}$, washed with water and dried $\left(\mathrm{MgSO}_{4}\right)$ to give 7 (70 mg, 88\%). ${ }^{1} \mathrm{H}$ NMR $\left(300 \mathrm{MHz}, \mathrm{CDCl}_{3}\right): \delta 7.45-7.26(\mathrm{~m}, 15 \mathrm{H}), 5.52$ (br s, $\left.1 \mathrm{H}\right), 5.11(\mathrm{~s}$, 2H), 4.91-4.80 (m, 3H), $4.65(\mathrm{~d}, J=10.8 \mathrm{~Hz}, 1 \mathrm{H}), 4.29(\mathrm{~d}, J=7.2 \mathrm{~Hz}, 1 \mathrm{H}), 3.93-3.84(\mathrm{~m}, 2 \mathrm{H}), 3.72-$ $3.70(\mathrm{~m}, 3 \mathrm{H}), 3.62-3.56(\mathrm{~m}, 2 \mathrm{H}), 3.47-3.30(\mathrm{~m}, 3 \mathrm{H}), 3.19(\mathrm{~m}, 2 \mathrm{H}), 3.00(\mathrm{~m}, 2 \mathrm{H}), 1.90-1.53(\mathrm{~m}, 6 \mathrm{H})$. ESI MS: $m / z$ : calcd for $\mathrm{C}_{35} \mathrm{H}_{48} \mathrm{O}_{9} \mathrm{~N}_{5} \mathrm{~S}: 714.3$; found: $714.2\left[\mathrm{M}+\mathrm{NH}_{4}\right]^{+}$.

Compound 7 (24 mg, $34 \mu \mathrm{mol})$ was dissolved in THF (4 mL) and treated with an aqueous solution of $\mathrm{NaOH}(0.1 \mathrm{~N}, 0.5 \mathrm{~mL})$. A solution of $\mathrm{PMe}_{3}$ in THF $(69 \mu \mathrm{L}$ of a $1 \mathrm{M}$ solution) was added and the reaction was stirred for $3 \mathrm{~h}$ at room temperature. The reaction mixture was neutralized with $0.1 \mathrm{~N}$ solution of $\mathrm{HCl}$, concentrated and the residue was eluted from a Sephadex LH-20 chromatography column with $\mathrm{MeOH} / \mathrm{CH}_{2} \mathrm{Cl}_{2}$ (1:1) to afford 8 (22 mg, 96\%). ${ }^{1} \mathrm{H}$ NMR (300 MHz, $\left.\mathrm{CD}_{3} \mathrm{OD}\right): \delta$ 7.33-7.22 $(\mathrm{m}, 15 \mathrm{H}), 5.08(\mathrm{~s}, 2 \mathrm{H}), 4.95-4.68(\mathrm{~m}, 4 \mathrm{H}), 4.45(\mathrm{~d}, J=8.1 \mathrm{~Hz}, 1 \mathrm{H}), 3.95-3.85(\mathrm{~m}, 2 \mathrm{H}), 3.78-3.56(\mathrm{~m}$ 6H), $3.43(\mathrm{~m}, 1 \mathrm{H}), 3.34-3.24(\mathrm{~m}, 2 \mathrm{H}), 3.12-3.07(\mathrm{~m}, 2 \mathrm{H}), 2.89(\mathrm{~m}, 1 \mathrm{H}), 1.88-1.51(\mathrm{~m}, 6 \mathrm{H})$. ESI MS: $m / z$ : calcd for $\mathrm{C}_{35} \mathrm{H}_{47} \mathrm{O}_{9} \mathrm{~N}_{2} \mathrm{~S}: 671.3$; found: $671.1[\mathrm{M}+\mathrm{H}]^{+}$.

To a solution of $\mathbf{8}(62 \mathrm{mg}, 92 \mu \mathrm{mol})$ in anhydrous pyridine $(2 \mathrm{~mL})$ was added sulphur trioxidepyridine complex (146 mg, $0.92 \mathrm{mmol})$. After stirring for $2 \mathrm{~h}$ at room temperature under an argon atmosphere, the reaction mixture was quenched with triethylamine $(0.1 \mathrm{~mL})$, stirred for $1 \mathrm{~h}$ and diluted with $\mathrm{MeOH}(1 \mathrm{~mL})$ and $\mathrm{CH}_{2} \mathrm{Cl}_{2}(1 \mathrm{~mL})$. The solution was layered on the top of a Sephadex LH-20 chromatograpy column that was eluted with $\mathrm{MeOH} / \mathrm{CH}_{2} \mathrm{Cl}_{2}(1: 1)$. The fractions that contained the sulphated monosaccharide were pooled and evaporated to dryness. The residue was converted into the sodium salt by elution from a column of Dowex 50WX4-Na ${ }^{+}$with $\mathrm{MeOH} / \mathrm{H}_{2} \mathrm{O}(9: 1)$ to give 17 (68 mg, 84\%). ${ }^{1} \mathrm{H}$ NMR (300 MHz, $\left.\mathrm{CD}_{3} \mathrm{OD}\right): \delta 7.43-7.17(\mathrm{~m}, 15 \mathrm{H})$, 5.12-5.08 (m, 3H), 4.76-4.72 (m, 4H), 4.33$4.22(\mathrm{~m}, 2 \mathrm{H}), 3.98(\mathrm{dd}, J=8.1,7.8 \mathrm{~Hz}, 1 \mathrm{H}), 3.88(\mathrm{~m}, 1 \mathrm{H}), 3.70-3.58(\mathrm{~m}, 5 \mathrm{H}), 3.34-3.22(\mathrm{~m}, 3 \mathrm{H}), 3.13-$ 
$3.09(\mathrm{~m}, 2 \mathrm{H}), 1.81-1.55(\mathrm{~m}, 6 \mathrm{H})$. ESI MS: $m / z$ : calcd for $\mathrm{C}_{35} \mathrm{H}_{45} \mathrm{O}_{15} \mathrm{~N}_{2} \mathrm{~S}_{3}: 829.2$; found: 828.9 [M$2 \mathrm{Na}+\mathrm{H}]^{-}$.

A solution of $17(66 \mathrm{mg}, 75 \mu \mathrm{mol})$ in $\mathrm{MeOH} / \mathrm{H}_{2} \mathrm{O}(4 \mathrm{~mL} / 2 \mathrm{~mL})$ was hydrogenated in the presence of $10 \% \mathrm{Pd} / \mathrm{C}$. After $24 \mathrm{~h}$, the suspension was filtered and concentrated to give 9 (41 mg, quantitative). $[\alpha]^{24}=-30.3\left(c=0.3, \mathrm{H}_{2} \mathrm{O}\right) ;{ }^{1} \mathrm{H}$ NMR $\left(300 \mathrm{MHz}, \mathrm{D}_{2} \mathrm{O}\right): \delta 4.44(\mathrm{~d}, J=8.1 \mathrm{~Hz}, 1 \mathrm{H}), 4.20(\mathrm{dd}, J=1.8$ $11.1 \mathrm{~Hz}, 1 \mathrm{H}), 4.06(\mathrm{dd}, J=5.4,11.1 \mathrm{~Hz}, 1 \mathrm{H}), 3.76$ (m, 1H), 3.60-3.48 (m, 3H), 3.37-3.31 (m, 3H), 3.20$3.14(\mathrm{~m}, 4 \mathrm{H}), 2.86(\mathrm{dd}, J=8.4,9.6 \mathrm{~Hz}, 1 \mathrm{H}), 1.76-1.40(\mathrm{~m}, 6 \mathrm{H}) .{ }^{13} \mathrm{C} \mathrm{NMR}\left(75 \mathrm{MHz}, \mathrm{D}_{2} \mathrm{O}\right): \delta 101.7$ 74.8, 73.6, 70.4, 69.8, 67.3, 60.2, 52.7, 51.9, 33.7, 28.3, 24.1, 20.9. ESI MS: $m / z$ : calcd for $\mathrm{C}_{13} \mathrm{H}_{26} \mathrm{O}_{13} \mathrm{~N}_{2} \mathrm{~S}_{3} \mathrm{Na}$ : 537.0; found: $537.0[\mathrm{M}-\mathrm{H}]^{-}$.

\section{Mass Spectrometry Data of Glycoconjugates 10, 11 and 12}

Glycoconjugates 10, 11 and 12 were synthesized by hydrogenolysis of the corresponding fully sulfated and benzylated compounds 18, 19 and 20 (Scheme 3), respectively, as described above for the preparation of monosaccharides $\mathbf{4}$ and $\mathbf{9}$.

Glycoconjugate 18: ES MS: $m / z$ : calcd for $\mathrm{C}_{48} \mathrm{H}_{59} \mathrm{O}_{24} \mathrm{~N}_{2} \mathrm{~S}_{4}$ : 1175.0; found: 1175.0 [M-4Na+3H]; calcd for $\mathrm{C}_{48} \mathrm{H}_{58} \mathrm{O}_{24} \mathrm{~N}_{2} \mathrm{~S}_{4}$ : 587.5; found: $587.0[\mathrm{M}-4 \mathrm{Na}+2 \mathrm{H}]^{-2}$.

Glycoconjugate 19: ES MS: $m / z$ : calcd for $\mathrm{C}_{74} \mathrm{H}_{88} \mathrm{O}_{43} \mathrm{~N}_{3} \mathrm{~S}_{7}$ : 643.3; found: 643.3 [M-8Na+5H] $]^{-3}$; calcd for $\mathrm{C}_{74} \mathrm{H}_{87} \mathrm{O}_{43} \mathrm{~N}_{3} \mathrm{~S}_{7} \mathrm{Na}$ : 650.7; found: $650.8[\mathrm{M}-7 \mathrm{Na}+4 \mathrm{H}]^{-3}$; calcd for $\mathrm{C}_{74} \mathrm{H}_{88} \mathrm{O}_{43} \mathrm{~N}_{3} \mathrm{~S}_{7} \mathrm{Na}$ : 976.5; found: $976.7[\mathrm{M}-7 \mathrm{Na}+5 \mathrm{H}]^{-2}$.

Glycoconjugate 20: ES MS: $m / z$ : calcd for $\mathrm{C}_{100} \mathrm{H}_{118} \mathrm{O}_{62} \mathrm{~N}_{4} \mathrm{~S}_{10}$ : 671.5; found: $671.6[\mathrm{M}-12 \mathrm{Na}+8 \mathrm{H}]^{-4}$.

Glycoconjugate 10: ES MS: $m / z$ : calcd for $\mathrm{C}_{19} \mathrm{H}_{34} \mathrm{O}_{22} \mathrm{~N}_{2} \mathrm{~S}_{4} \mathrm{Na}$ : 793.0; found: 792.8 [M-2Na+H]

Glycoconjugate 11: ES MS: $m / z$ : calcd for $\mathrm{C}_{31} \mathrm{H}_{52} \mathrm{O}_{41} \mathrm{~N}_{3} \mathrm{~S}_{7} \mathrm{Na}$ : 684.5 ; found: 684.5 [M-6Na+4H] $]^{-2}$; calcd for $\mathrm{C}_{31} \mathrm{H}_{51} \mathrm{O}_{41} \mathrm{~N}_{3} \mathrm{~S}_{7} \mathrm{Na}_{2}$ : 695.5; found: $695.5[\mathrm{M}-5 \mathrm{Na}+3 \mathrm{H}]^{-2}$.

Glycoconjugate 12: ES MS: $m / z$ : calcd for $\mathrm{C}_{43} \mathrm{H}_{70} \mathrm{O}_{60} \mathrm{~N}_{4} \mathrm{~S}_{10} \mathrm{Na}_{2}$ : 984.0; found: 984.0 [M-9Na+7H] $]^{-2}$; calcd for $\mathrm{C}_{43} \mathrm{H}_{68} \mathrm{O}_{60} \mathrm{~N}_{4} \mathrm{~S}_{10} \mathrm{Na}_{4}$ : 1006.0; found: 1006.0 [M-7Na+5H] $]^{-2}$; calcd for $\mathrm{C}_{43} \mathrm{H}_{70} \mathrm{O}_{60} \mathrm{~N}_{4} \mathrm{~S}_{10} \mathrm{Na}$ : 648.3; 
found: $648.4[\mathrm{M}-10 \mathrm{Na}+7 \mathrm{H}]^{-3}$.

Scheme 3. Synthesis of Glycoconjugates 10, 11 and 12.

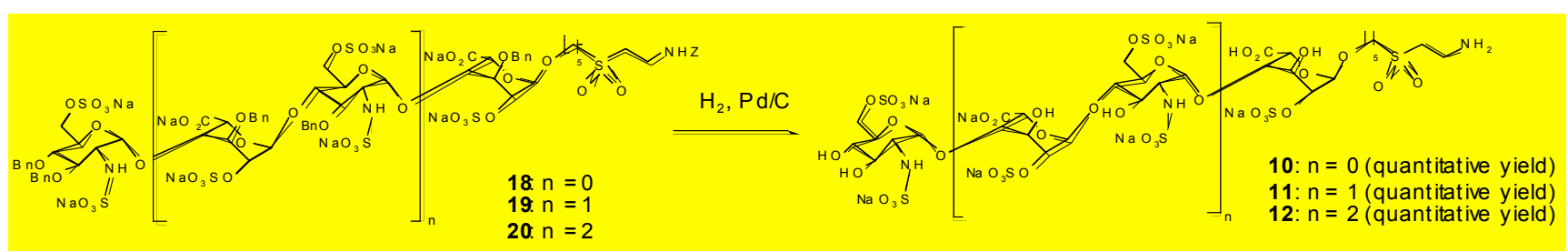

\section{Experimental Procedures for the Production of the Heparin Microarrays}

Immobilization of Heparin Oligosaccharides: The monosaccharides $\mathbf{4}$ and $\mathbf{9}$ as well as the heparin oligosaccharides 10, 11 and 12 along with 2'-aminoethyl-2-acetamido- $\alpha$-D-glucopyranoside (GlcNAc) and 2'-aminoethyl- $\beta$-D-galactopyranoside (Gal) were spatially arrayed onto CodeLink slides by use of an automated arraying robot (Perkin Elmer) in sodium phosphate buffer $(\mathrm{pH} 9.0,50 \mathrm{mM})$. The robot delivered $1 \mathrm{~nL}$ of sugar solutions at concentrations ranging from $2 \mathrm{mM}$ to $250 \mu \mathrm{M}$ and the resulting spots had a diameter of $\sim 200 \mu \mathrm{m}$ (500 $\mu \mathrm{m}$ spacing). Sodium phosphate buffer (pH 9.0, $50 \mathrm{mM}$ ) was used as a negative control and all samples were printed in replicates of fifteen (480 spots). After printing, slides were immediately placed in a humidity chamber and incubated for $12 \mathrm{~h}$. Slides were then washed three times with water to remove the unbound carbohydrates from the surface. Remaining succinimidyl groups were quenched by placing slides in a solution preheated to $50^{\circ} \mathrm{C}$ that contained 100 $\mathrm{mM}$ ethanolamine in sodium phosphate buffer $(\mathrm{pH} 9.0,50 \mathrm{mM})$ for $1 \mathrm{~h}^{4}$. Slides were rinsed several times with distilled water, dried by centrifugation and stored in a dessicator prior to use.

Array Incubation and Analysis: The FGF hybridization solutions were prepared by diluting the 
stock solutions to a concentration of $5 \mu \mathrm{g} / \mathrm{mL}$ with PBS buffer ( $\mathrm{pH} 7.5,10 \mathrm{mM})$ containing BSA (1\%). Array incubations were performed as follows: 60-80 $\mu \mathrm{L}$ of FGF solution were placed between array slides and plain coverslips and incubated for $1 \mathrm{~h}$ at room temperature. The arrays were washed with PBS (pH 7.5, $10 \mathrm{mM}$ ) containing $1 \%$ Tween 20 and $0.1 \%$ BSA, twice with water, and then centrifuged for 5 min to ensure dryness. For detection of bound FGF, arrays were incubated with anti-human FGF polyclonal antibody $(5 \mu \mathrm{g} / \mathrm{mL})$ and then washed as above. Finally, AlexaFluor-546-labelled anti-rabbit IgG was used as secondary antibody and again washed as above. Heparin arrays were scanned by using a LS400 scanner (Tecan) and fluorescence intensities from these scans were integrated on Scan Array Express Software (Perkin Elmer). Data presented are the average of 15 spots at $500 \mu \mathrm{M}$ (subsaturating level concentration) on the same array; errors are the standard deviations for each measurement (Figure 1).

Figure 1. Top: Microarray after Incubation with FGF-2. Bottom: Fluorescence Signal Observed for each Arrayed Carbohydrate Binding to FGF-2 at $500 \mu \mathrm{M}$.

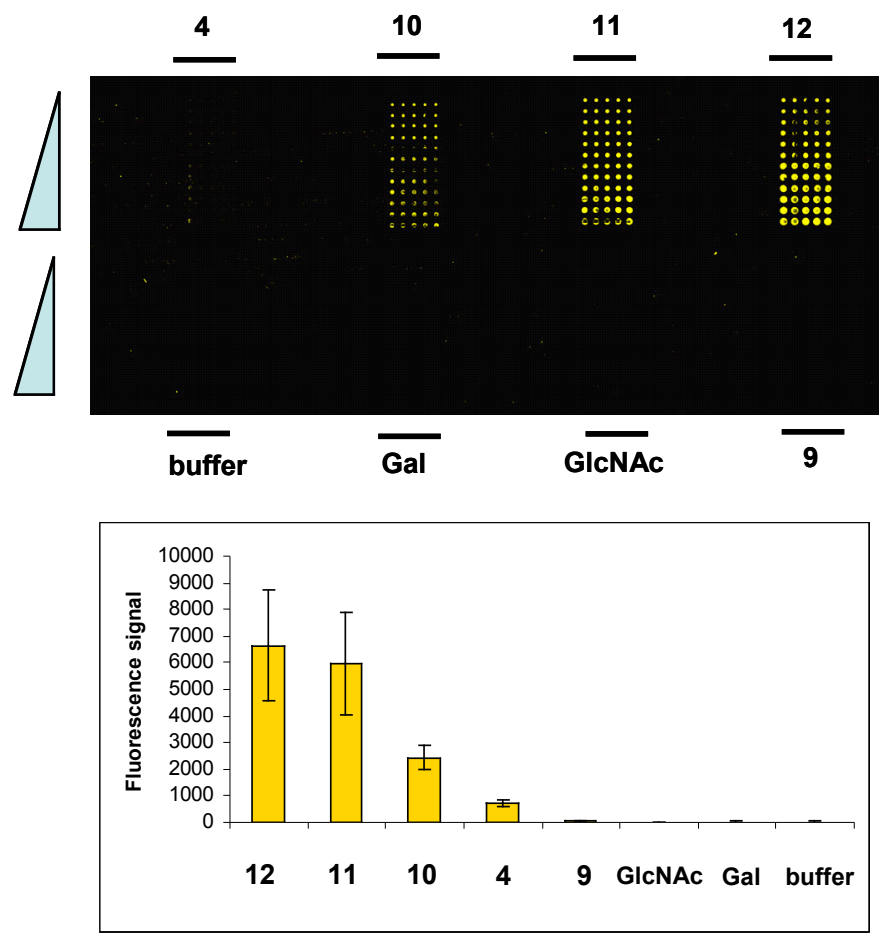




\section{Additional Negative Control for the Heparin Microarrays}

Monosaccharide 3 was submitted to hydrogenolysis, giving compound 21 (Scheme 4) that was used as an additional negative control for the heparin microarrays. Compound 21, that contains the same "sulfone" linker than monosaccharides $\mathbf{4}$ and $\mathbf{9}$ as well as the oligosaccharides 10-12, is useful to discard the participation of the linker in the binding events. Monosaccharides $\mathbf{4}$ and 21, as well as the hexasaccharide 12, were manually spotted on CodeLink slides at concentrations ranging from $5 \mathrm{mM}$ to $50 \mu \mathrm{M}$ (Figure 2). After incubation with FGF-1, strongly fluorescent signals were observed for compounds 4 and 12, while no signal was detected for the non-sulfated monosaccharide 21 even at high concentrations (5mM). Similar results were obtained after incubation with FGF-2 (data not shown).

Monosaccharide 21. ${ }^{1} \mathrm{H}$ NMR $\left(300 \mathrm{MHz}, \mathrm{D}_{2} \mathrm{O}\right): \delta 4.72(\mathrm{~d}, J=5.1 \mathrm{~Hz}, 1 \mathrm{H}), 4.37(\mathrm{~d}, J=4.8 \mathrm{~Hz}, 1 \mathrm{H})$, 3.82-3.79 (m, 2H), 3.67-3.61 (m, 3H), $3.53(\mathrm{~m}, 2 \mathrm{H}), 3.43-3.38(\mathrm{~m}, 3 \mathrm{H}), 3.29(\mathrm{~m}, 1 \mathrm{H}), 1.89-1.52(\mathrm{~m}$, 6H). ESI MS: $m / z$ : calcd for $\mathrm{C}_{13} \mathrm{H}_{26} \mathrm{O}_{9} \mathrm{NS}: 372.1$; found: $372.0[\mathrm{M}+\mathrm{H}]^{+}$.

Scheme 4. Synthesis of Monosaccharide 21

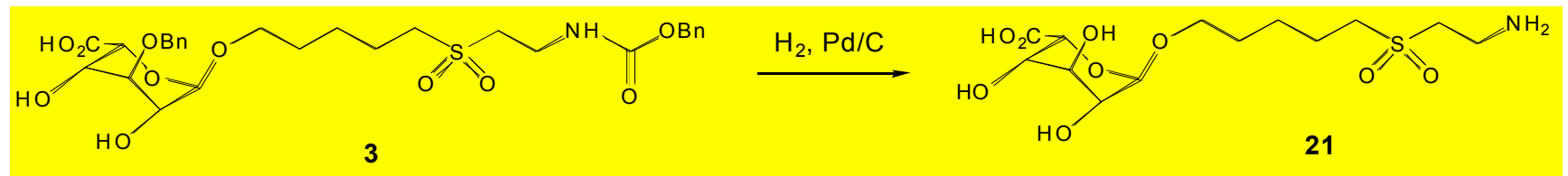

Figure 2. Microarray after incubation with FGF-1.

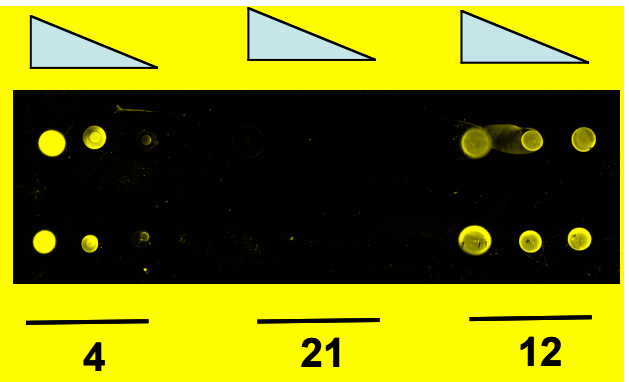

\section{Additional References.}

1. Ojeda, R.; De Paz, J. L.; Martin-Lomas, M.; Lassaletta, J. M. Synlett 1999, 1316-1318. 
2. Shinkai, I.; Liu, T.; Reamer, R.; Sletzinger, M. Synthesis 1980, 924-926.

3. Orgueira, H. A.; Bartolozzi, A.; Schell, P.; Litjens, R. E. J. N.; Palmacci, E. R.; Seeberger, P. H. Chem. Eur. J. 2003, 9, 140-169.

4. Disney, M. D.; Seeberger, P. H. Chem. Biol. 2004, 11, 1701-1707. 